This document is the Accepted Manuscript version of the following article:

Brendan Larvor, 'How to think about informal proofs', Synthese, Vol. 187(2):

715-730, first published online 9 September 2011.

The final publication is available at Springer via doi:10.1007/s11229-011-0007-5 


\section{How to think about informal proofs}

Abstract: It is argued in this paper that (i) progress in the philosophy of mathematical practice requires a general positive account of informal proof; (ii) the best candidate is to think of informal proofs as arguments that depend on their content as well as their logical form; (iii) articulating the dependency of informal inferences on their content requires a redefinition of logic as the general study of inferential actions; (iv) it is a decisive advantage of this conception of logic that it accommodates the many mathematical proofs that include actions on objects other than propositions; (v) this conception of logic permits the articulation of project-sized tasks for the philosophy of mathematical practice, thereby supplying a partial characterisation of normal research in the field.

The philosophy of mathematical practice prides itself on paying attention to the proofs that mathematicians offer each other, rather than the abstract models of proofs studied in formal logic. This is the mathematical version of the boast of informal logic and argumentation theory, to study the arguments that people actually make-'real' arguments (as in the titles of Corfield 2003 and Fisher 2004). The philosophy of mathematical practice has now acquired a body of literature (leading examples in addition to Corfield 2003 being Aspray and Kitcher, 1988; Buldt et al., 2008; Cellucci and Gillies, 2005; Grosholz and Breger, 2000; Hanna et al., 2010; Hersh, 2006; Lakatos 1976; Loewe and Mueller, 2010; Mancosu 2008; Nabonnand, 2005; Van Kerkhove, 2009; Van Kerkhove and Van Bendegem, 2002, 2007) and a freshly constituted international Association for the Philosophy of Mathematical Practice. However, it remains somewhat under-theorised. Answers to the questions 'What is the philosophy of mathematical practice?' and 'How does one do it?' do not usually go far beyond the aspiration to study 'actual' mathematical activity and some now 
familiar complaints about other, better-established approaches to the philosophy of mathematics that employ formal models of mathematics and mathematical argument. Among other things, the field lacks an explication of 'informal proof' as it appears in expressions such as 'the informal proofs that mathematicians actually read and write'. Without this, it is difficult to explain how studies of practice might diagnose and overcome the short-comings of those approaches that take formal logic to supply an adequate account of mathematical inference. This shows up practically as a methodological gap. It is not yet clear how the historical, sociological and psychological studies presented at conferences on the philosophy of mathematical practice can generate a significant challenge to the approaches that assume that formal logic can provide a philosophically adequate model of mathematical proof.

Thus, the logic of informal proofs matters for two reasons. First, the complaints about the 'traditional' approaches to the philosophy of mathematics need some theory to turn them into robust objections. Philosophers of mathematical practice disagree among themselves about the logical relation between the philosophy of mathematical practice and other traditions in the philosophy of mathematics. ${ }^{1}$ Even the most ecumenical philosopher of mathematical practice must convict the other traditions of at least a sin of omission, namely, failing to pay attention to mathematical practice. However, we require an argument to show that (and why) this omission is a sin. After all, the rival traditions have replies available. They can point out that all models are idealisations, that philosophy always abstracts from particulars, and that it is a philosophical virtue to discard inessentials. They might continue thus: the essence of proof is the notion of logical consequence, which formal logic models precisely; therefore formal logic is just the right tool for the

\footnotetext{
${ }^{1}$ Mancosu sees philosophical attention to mathematical practice as a welcome and compatible addition to the previously established traditions (editorial introduction in 2008); Corfield claims that mainstream philosophy of mathematics is incompatible with a practice-based approach because it imposes a 'foundationalist filter' that blinds it to real mathematical practice (2003 p. 8).
} 
philosophical study of mathematical argument. Philosophers of mathematical practice need to show that mathematical arguments suffer some philosophically important loss or distortion in the abstraction from 'real' mathematical proof to formal derivation. For the loss or distortion to be philosophically interesting, it must have some logical significance. Whatever gets lost or distorted must play a role in the account of how informal proofs work as proofs. Otherwise, opponents of practice-based philosophy can safely park the results of psychological, sociological and historical studies on the 'discovery' side of the discovery/justification distinction. To counter this, philosophers of mathematical practice need a conception of argument that permits them to say of (some of) their studies of mathematical practice that this, too, is logic.

Second, the philosophy of mathematical practice has yet to establish an understanding of normal research in its field. In spite of the growing literature, we do not have many exemplars for research students to emulate. We aim to study 'what mathematicians actually do', yes, but some of the things they 'actually' do (such as drinking coffee or going for solitary walks) probably are philosophically irrelevant. Other activities, such as drawing diagrams, refining mathematical models and (of course) creating proofs, clearly are philosophically interesting parts of mathematical practice. Then there are activities that involve reasoning about mathematics, such as refereeing papers, judging $\mathrm{PhDs}$ and awarding prizes, that occupy a grey area. In the absence of some background philosophical theory, it is hard to judge which of the mathematicians' actual activities should be philosophically salient. The most influential works in the philosophy of mathematical practice are often too singular to suggest themselves as models. There would (for example) be little merit in mass production of fictional dialogues with historical footnotes. For this reason too, the philosophy of mathematical practice needs to specify what is philosophically important about mathematical practice, as represented by 'real' mathematical proofs, that is absent from derivations in formal logic. 


\section{Essentially Informal Arguments: definition and problem}

Happily, there is no difficulty identifying informal proofs. There is a via negativa, thus: formal arguments (a) are expressed in a general logical language, the well-formed formulae of which are explicitly defined (usually by recursion) and (b) consist of successive applications of explicitly specified rules of logical inference (in some systems some of these may be expressed as logical axioms). Informal arguments are all the others. Notice that a mathematical proof may employ little or no natural language, make no appeal to spatial or arithmetical intuition and proceed from explicit definitions and/or axioms, yet still not satisfy (a) and (b) and therefore count as informal. Almost all mathematical proofs are informal by this standard, including the proofs published in research mathematics journals. No-one disputes this. Nor is it disputed that many informal proofs can provide source material for formal derivations. The problem before philosophers of mathematical practice is to identify and characterise those informal arguments that would suffer some sort of violence or essential loss if they were recast so as to satisfy (a) and (b). Call these 'essentially informal' arguments. With this terminology, we can express the distinctive conviction of the philosophy of mathematical practice on the subject of proof thus: almost all of the informal proofs that mathematicians actually read, write and publish are essentially informal. Now, the negative definition of informal proofs as 'those proofs that fail to satisfy at least one of (a) and (b)' leaves open the possibility that all informal mathematical arguments could be fully formalised without loss or violence. What we need is a positive account of the notion of an essentially informal argument. Formal logic cannot supply this. Where else might we look?

\section{Argumentation Theory and Informal Logic}

Since the central claim of the philosophy of mathematical practice - the focus on 'real' proofs_-is the mathematical case of the chief merit claimed for argumentation theory, one might expect that the latter could supply some theory and method to the former. Alas, argumentation theory is less 
helpful than one might have hoped, because it assumes (almost without exception) that arguments are made of speech-acts. This is not unreasonable, since almost all argument (including, obviously, philosophical argument) happens in and with spoken or written language. The central claim of this paper is that in an important sense this is not true of most mathematical argument. In a slogan: inferential acts are not always speech-acts. Before considering the case for this claim, it will be useful to make some orienting remarks about argumentation theory and informal logic.

One strong current in contemporary argumentation theory is a tendency to distinguish real arguments from the artefacts of formal logic by pointing to the embeddedness of real arguments in practical contexts. Real arguments are the arguments that real people address to other real people in real situations to try to achieve real ends. Here is a typical statement from apostate formal logician Don Levi:

To determine [using formal logic] whether something is being argued or what the argument is, and to evaluate it, the speaker's words are supposed to be restated in premise-conclusion form. This restatement leads to the neglect of the rhetorical context - the argument in that form no longer has an audience, or for that matter a speaker, whose concerns help to explain what she is arguing. ${ }^{2}$

In other words, the argumentation theorist of this stripe denies that the argument-as-such can be lifted out of its argumentative context and evaluated 'in purely logical terms'. Such an act of abstraction violently severs the connections that make the argument intelligible. Here is a more technical version of the same thought, in the words of two of the leading voices in the field:

Argumentation is adduced in reaction to, or in anticipation of, a difference of opinion, and serves a role in the regulation of disagreement. Not only the need for argumentation, but also its internal and external structure and the criteria that

2 Levi (2010) pp. 80-81. 
it must meet, are directly related to the doubt or criticism that the argumentation is intended to remove. ${ }^{3}$

It's true that argumentation is sometimes used to regulate and resolve differences of opinion. But is it always?

The history of mathematics (and perhaps some other disciplines ${ }^{4}$ ) suggests not. Certainly, all (real) mathematical arguments have contexts. Proofs are tuned to and pitched at particular intended audiences. They have dense intertextual relations with prior works (in addition to citing previously established results as lemmas, a proof may depend on prior works to show that it answers a pressing question, contributes to a coherent body of knowledge, adopts reasonable conventions, promises further illumination either as corollaries or by the repeated use of its characteristic techniques, etc.). Proofs are conceived in, born into and achieve maturity in richly specific cultural and historical locations. It is entirely proper and worthwhile to study this. ${ }^{5}$ This approach is particularly relevant for mathematics education (see CadwalladerOlsker 2011). However, mathematical arguments tend to develop careers beyond the persuasive jobs for which they are first invented. Few (if any) readers of Euclid's Elements know whom Euclid was trying to convince or what specific 'doubt or criticism ' he was trying to remove. Indeed, it is not clear that removing doubt or criticism was ever the purpose of these proofs. ${ }^{6}$ They (and mathematical proofs in general) can be understood without reconstructing the dialogical structure of their argumentative contexts. For this reason, references

${ }^{3}$ Eemeren, F.H. van, \& Grootendorst, R. (2004) p. 53. Since Grootendorst's death in 2000, Eemeren has gone further in this direction, writing several studies of 'strategic manoeuvring'.

4 Andrew Aberdein made this plausible suggestion.

${ }^{5}$ Andrew Aberdein, for example, explores the dialogical nature of mathematical proof in (Aberdein 2006).

6 Which is not to say that the logic of Euclid's arguments is obvious or easy to make explicit. We do need to know a lot about (for example) how the ancient Greeks thought about number. We do not need to know what doubts and criticisms motivated the production of the Elements. See Manders (1995) 
to argumentative context cannot help us to unpack the meaning of 'informal' in phrases such as 'the informal proofs that mathematicians actually read and write'. ${ }^{7}$

If the notion of dialogical context does not supply a robust account of essentially informal proofs, then what other options are available? There are some other candidate-accounts for the distinction between formal and informal arguments that we can dispose of quickly, without having to ask whether they offer any insight into mathematics. First, valid formal arguments are not ampliative, so one might imagine that this is the vital difference. However, there are non-ampliative arguments that seem to be essentially informal. For example, arguments from authority such as 'This person is an expert on tax law and he says that you are liable in such and such a respect, therefore, you are so liable' do not have anything in the conclusion that is not already present in the premises. Moreover, neither informal mathematical proofs nor formal derivations are ampliative, so the ampliative/nonampliative distinction cannot articulate the difference between them. Second, one might think that the sought-after distinction is between deduction and induction. However, not all informal arguments are inductive, in any strict sense of the term 'induction'. In fact, relatively few informal arguments proceed from a common property of a lot of particulars to the claim that one or more particulars not yet mentioned share that same property. Besides, we may doubt that strictly inductive arguments are essentially informal. Turning to mathematics, neither informal mathematical proofs nor formal derivations are strictly inductive, so the inductive/deductive distinction is no help in the project of grounding the philosophy of mathematical practice. (Which is not to say that enumerative induction has no place in mathematical reasoning. See Baker in Leng et al. (2007); Gowers op. cit. pp. 34-5; and Corfield (2003) chapter 5.)

\footnotetext{
Azzouni (2006) develops the claim that mathematics establishes beliefs and practices that last much longer than any social structures or other social phenomena. Mathematics cannot be a social construction, Azzouni argues, because social constructions don't last as long as theorems (even if we think of theoremhood as a social-historical category).
} 
Another powerful stream in argumentation theory is the exploration of argumentation schemes. Here, the ambition is to develop a taxonomy and ultimately a general theory of defeasible argument schemata such as analogy, appeals to expertise and testimony and the various kinds of slippery slope. The quest for a taxonomy and general theory means that this approach abstracts from both the dialogical context and the content of the argument. The most developed product of this research programme, Walton, Reed and Macagno (2008), has as its penultimate chapter a discussion of the work to date on formalising argument schemes, and as its final chapter a survey of argument schemes in computer systems. This version of the argument schemes research programme is not a rival to the project of analysing all argument formally. It is, rather, the extension of that project to include non-inductive defeasible argument. As such, it has to reject the claim that the arguments it analyses are essentially informal. Moreover, as the final chapters of Walton, et al. illustrates, it substitutes a disembodied ideal reasoner for the embodied human arguer. For these reasons, it is unsuited to the project of understanding how embodied, human mathematicians use essentially informal proofs to establish theorems. ${ }^{8}$ Of course, Walton, et al. do not have exclusive ownership of the argumentation schemes idea. It is possible to use it without attempting to formalise argumentation schemes. However, to make a virtue of this, one would have to answer our question: what is an informal argument?

\section{Essentially Informal Proofs: Solution}

A more plausible account of essentially informal arguments, and the one that this paper will take up and develop, claims that the validity or invalidity of essentially informal arguments does not depend on their logical form alone, but also on their content - they are content-dependent. ${ }^{9}$ For example, an appeal to authority may or may not be a good argument, depending on which expert is appealed

\footnotetext{
${ }^{8}$ For more on embodiment in mathematics, see Johansen (2010). See Van Kerkhove, B.; Van Bendegem, J.-P. (2009) for an extended discussion of the application of argument schemes to mathematics.

9 Lakatos glosses 'informal' in 'informal mathematics' as 'inhaltliche' (i.e. having or concerning content) (1976:1)
} 
to and on what question. Aside from the credentials of this or that expert, note that arguments of this sort require that the subject-matter be such as permits expertise. (A poor argument: 'The Ferrari will win. For, X says so, and he is an expert on loud things'. Loud things do not constitute a possible field of expertise. The behaviour of jet aircraft is not systematically related to the behaviour of rock bands.) To change the example, for a slippery-slope argument to succeed, there must be a slope, it really must be slippery and there must be a force drawing the argument down the slope. Determining whether these conditions obtain requires examination of the argument's content. The relevant point for this paper is that slippery-slope arguments can work only in domains with candidates to play the roles of gradation, gravity and grease. This is the thought that I want to carry into the mathematical case: that essentially informal arguments are content-dependent partly because they require domains with suitable general features. The presence of such features does not guarantee that an argument is sound. Rather, they are the necessary conditions for attempting to make an argument of that sort at all. Note, these argument patterns are not strictly domain-specific, because they can work in more than one domain (we appeal to authority on all sorts of topics and there are slopes in many domains). The point is that unlike, say, modus ponens, they do not work in all domains. Modus ponens may be expressed informally, but it is not essentially informal. It does not depend on its content, that is, it applies in all domains, which is why it can be rewritten to satisfy (a) and (b) without loss or distortion. Its topic-neutrality means that it can be captured in a purely logical language, as (a) requires.

This thought, that essentially informal arguments depend for their inferential power on their content as well as their forms, requires a shift in how we think about arguments. If we think of an argument as a sequence of propositions connected by logical relations, it is hard to see how the content of the argument can play a role in the step from one proposition to the next. This is in part because a classically trained philosophical imagination is dominated by general logic, but also because 
orthodox philosophical education urges us to forget that the movement from one line of a proof to the next is an action. The standard view, usually associated (perhaps unfairly) with Frege, is that logic must be purged of the inferring agent, or else fall into psychologism. Nevertheless, the rules of inference coded in formal systems are procedures, that is, standardised actions. Proving is an activity, even when the results are mechanically checkable. The liberating insight is to notice that in making arguments, we act on all sorts of items in addition to propositions and well-formed formulae. Sometimes, we act inferentially on non-propositional representations of the subjectmatter such as diagrams, notational expressions, physical models, mental models and computer models. Sometimes, we re-describe the subject-matter in some insightful way, perhaps with the aid of an analogy or metaphor. Sometimes, we act directly on the subject-matter itself, taking measurements or performing physical experiments. Sometimes, we perform thought-experiments and calculations, or show that something exists by manufacturing it (as in the 'search' for sub atomic particles). Sometimes, we act on experts by asking them questions. Some inferential actions do not have objects; for example, one might show that an unlikely act (such as a new move in gymnastics) is possible by performing it. Many of these actions are repeatable procedures, and learning to carry them out is part of the training of new experts. Every successful calculus student learns how to change variables, and every competent accountant learns how to draw up a balance sheet. On the other hand, very few of these actions are possible in all domains. In summary: the subject-matter of an argument can play a role in the argument's inferences if it (the subject-matter) is the object of an inferential action (for example, physical experiments); if a representation of it is the object of an inferential action (for example, manipulation of models); or if it is manifested or expressed in an inferential action (the case of the new gymnastic feat). Arguments that involve such inferences are essentially informal; they cannot be fully formalised in a general logic. 


\section{Inferential Acts Are Not Always Speech Acts}

Curiously, this insight, that argument is not all about propositions, is largely absent from the informal logic literature, even though it suggests an account of what informal arguments are. Typically, both textbooks and theoretical works on informal logic assume that arguments are made of statements ${ }^{10}$ and in doing so leave open the possibility that informal arguments are simply arguments that could be formalised but are not for some practical reason. To pick some examples arbitrarily, Beardsley explains that "to reason is just to take one statement as a reason for another" (1950 p. 9). In fact we do not reason from statements alone. One might, for example, take the smell of toast as a reason to think that someone is making breakfast. The first six out of Beardsley's fifteen chapters are about language. Similarly, Fogelin and Sinnott-Armstrong announce on page one that, "Arguing is... a linguistic activity" (italics in original) and that "Arguments are constructed out of sentences" (2001). They do move in the general direction of this paper by treating arguments as speech-acts (pp. 46 7). We need only add that arguments are indeed acts, but not always speechacts. One might demonstrate that a well is deep by dropping a stone into it and staring pointedly at one's wristwatch until hearing the splash, without uttering a word. While this action might be thought to have some illocutionary force, it is not best thought of as a speech-act because there is no wholly linguistic performance that could substitute for the physical experiment. Pace Derrida, il ya un hors-texte. Scriven, in his textbook, is more liberal: "Reasoning isn't all done with language," but having opened the door, immediately seeks to close it, “...but that's how it's usually conveyed and mostly how it's taught, and certainly how it's written and thus best recorded" (1976 p. 3). This might come as news to anyone who conveys, teaches and records reasoning with diagrams, models or recordings of experiments. To be fair, on the next-but-one page, Scriven notes that reasoning sometimes involves such activities as calculating, measuring and appealing to authority. He is

\footnotetext{
${ }^{10}$ See Hoagland (1999) for an overview.
} 
right, of course, that much reasoning is carried out in natural language; it often is the best medium for inferential actions such as drawing analogies or recalling precedents (and deploying philosophically interesting examples). If one has (say) law or philosophy in mind, it is easy to fall into thinking that inferential acts are all speech-acts.

Turning from textbooks to theoretical works, Eemeren \& Grootendorst announce that, "In principle, argumentation is a verbal activity, which takes place by means of language use..." (italic in original) (2004 pp. 1-2). They add in a footnote: "In practice, argumentation can also be partly, or even wholly, non-verbal... this is not adverse to our pragma-dialectical approach as long as the (constellation of propositions constituting the) argumentation is externalizable.” (p. 2n2). In keeping with this, studies of visual argumentation in this tradition normally treat pictures and diagrams as alternative presentations of propositional content. ${ }^{11}$ This does not leave space for the thought that (for example) a geometrical diagram is the object of geometrical inferential actions. As already noted, the other major stream in argumentation theory is the categorisation and formalisation of argumentation schemes. These schemes relate propositions as premises and conclusions, just as mainstream logical theory has since Aristotle. Because they model defeasible arguments, they include critical questions as well as statements, but these do not open space for any actions other than speech-acts..

Thinking of arguments as acts, and widening the scope of logic to include inferential actions in addition to those performed on propositions does not invite subjectivism in any worrying sense. There is an actor for every action, but for logical purposes we do not need to know anything about the agent beyond the fact that he or she performed this act. The whole person may be phenomenologically implicit in the act, but considering it as an inferential action permits us to bracket the agent (except in special cases such as appeals to expertise or pro homine arguments).

\footnotetext{
${ }^{11}$ See, for example, Groarke (1999)
} 
For an analysis of a proof into a series of inferential actions to have any plausibility, the actions must be feasible for a human actor, but this need not detract from their inferential power.

The benefit of viewing inference as action is that we can see how the subject-matter of informal arguments shapes and contributes to inferences. Indeed, instead of two highly abstract categories, the form of an argument and its content, we now have an indicative list of many and various concrete objects of inferential action (diagrams, models, expressions in special notations, experimental set-ups and so forth). This goes some way towards answering (or at least, making more precise) our question about which activities to count as mathematical practice. The cost is that we have to abandon the hope of establishing a general test for validity. It may be possible to automate some procedures (such as dendrochronology); for others (such as changing variables) it is possible to run automatic checks, but this will not be possible for all informal arguments. In any case, there is no hope of anticipating the invention of new inferential actions. This is one reason, and a respectable reason at that, for trying to re-describe all inferential action as action on propositions. Deductive formal logic is a partial model of the inferential actions possible on propositions, and it offers a criterion for identifying which actions are permissible. Twentiethcentury philosophy of science made heroic attempts to develop similar models for scientific reasoning, but the restriction of inferential actions to actions on propositions resulted in philosophers treating experiments as black boxes that emit 'observation statements' or Protokollsätze. Studies of experimental practice have since ${ }^{12}$ revealed that there is reasoning going on inside the experimental box, but in order to understand it, we have to recognise inferential actions on a wider range of objects than propositions alone. (Similarly, one might reconstruct the stone-dropping argument for the depth of a well as a sequence of statements, starting with the statement that when a stone was dropped, there was an interval of $x$ seconds before a splash was

12 For example, Galison $(1987 ;$ 1997). Versions of this point also occur in works by W. Whewell, N.R. Hanson, T.S. Kuhn and subsequent contributors to the debate on the 'theory-ladeness of data'. 
heard, but this would have the perverse consequence of leaving the central act of the argument, the dropping of the stone, out of the reconstruction, and would obscure the argument's tacit appeal to the embodied natures of both the arguer and the viewer, the reliability of their sense organs, the transparent familiarity of life in the gravitational and atmospheric conditions of the surface of the Earth, the trustworthiness and competence of the experimenter, and so forth.)

This section started out talking vaguely about informal arguments suffering distortion or loss when formalised. We are now in a position to tidy this up. A fully formal derivation (one that satisfies (a) and (b) above) is a sequence of actions performed on well-formed formulae expressed in a logical language. These actions produce new, well-formed combinations of non-logical expressions (the names, variables, atomic propositional letters, predicates and relations), but they do not change the non-logical expressions. Therefore, if an argument includes an inferential action that manifests or manipulates the subject-matter, or a representation thereof, then formalising this argument in a general logical language must either misrepresent or fail to include this action. Moreover, we can say something in the direction of explaining how informal arguments work as arguments: they are rigorous if they conform to the controls on permissible actions in that domain. An action demonstrating (by performance) the possibility of a new gymnastic feat had better conform to the rules of gymnastics; ice-core samples must be kept free of contamination; and so on.

\section{Philosophy of Mathematical Practice: the State of Play}

As noted at the outset, philosophers of mathematical practice like to insist on the differences between 'real' informal mathematical proofs and the formal derivations studied in the branch of mathematical logic called 'proof theory'. All parties acknowledge these differences. The debate arises out of the fact that the informal proofs used by expert mathematicians are highly compressed abbreviations of mathematical arguments. As Russell and Whitehead observed, "Most mathematical investigation is concerned not with the analysis of the complete process of reasoning 
but with the presentation of such an abstract of the proof as is sufficient to convince a properly instructed mind." ${ }^{13}$ So what would the fully-explicated 'complete process of reasoning' look like? Would it be a formal derivation? Saunders Mac Lane, reflecting on mathematical rigour, claimed that, "In practice, a proof is a sketch, in sufficient detail to make possible a routine translation of this sketch into a formal proof." (1986 p. 377). By 'formal proof', Mac Lane means a proof that is not content-dependent: “...the test for the correctness of a proposed proof is by formal criteria and not by reference to the subject matter at issue" (1986 p. 378; emphasis added). However, the proofs that mathematicians create and deploy typically make inferences that exploit local features of the subject-matter in hand. Euclid's proof of the infinitude of primes employs the fact that if a natural number $\mathrm{m}(>1)$ divides another, $\mathrm{n}$, it cannot divide $\mathrm{n}+1$. In this proof, this fact licences an argument-pattern or mini-method, namely, the trick of adding one to the factorial of some number $\mathrm{n}$ and then noticing that $n !+1$ cannot share any divisors with $n !$. This trick has been used in proofs of other theorems. This portability makes it logic-like, in the sense that it could be used in proofs of indefinitely many theorems, but it is not wholly general, because its use is restricted to subjectmatters of the right sort. The strategy of Euclid's proof employs a construction that works for numbers but not for continuous magnitudes, and which again has applications in other proofs (Rav calls it a 'topic-specific move' 1999 p. 26).

To cite another (albeit related) example, Goldbach's 1730 proof of the infinitude of primes exploits a feature of Fermat numbers: any two Fermat numbers are relatively prime. This is easy to prove from a recursive definition that is itself easy to verify by induction. Mathematical induction is not a general rule of inference like modus ponens; it works only where the subject-matter can be indexed to the natural numbers. Mathematical proofs typically use tricks, strategies, methods and devices that have some application beyond the proof in hand, but are not wholly general, in that they only

13 Whitehead and Russell (1962) p. 3-introduction to the first edition. 
work in domains of the right sort. ${ }^{14}$ These proof-ideas are thus content-dependent in something like the way that essentially informal argument patterns are.

Of course, Mac Lane recognised that proofs do more than secure the truth of their theorems. He appeals to this fact to explain why mathematicians do not write out derivations in full. They content themselves with the sketches and recipes because, "proofs are not only a means to certainty, but also a means to understanding. Behind each substantial formal proof there lies an idea... it will not do to bury the idea under the formalism." (ibid.). Mac Lane does not say what the proof-idea helps us to understand; presumably either the theorem or the working of the proof. In either case, the proof-idea is related in some intimate way to the content. Either it explains the truth of just that particular theorem, or it is a proof-strategy that works for some limited range of proof-types. When we combine the content-dependency of proof-ideas with Mac Lane's view that proofs should be tested by formal criteria only, we get the odd result that an informal proof is a content-dependent recipe for producing a content-independent derivation, and the (informal) proof-idea expressed in this proof should help us to understand something about the content, even though the content plays no role in the inferences that jointly constitute the (fully-explicated) proof.

Philosophers of mathematical practice have had plenty to say about the short-comings of the view that 'real' proofs are sketches of derivations. One of the lessons of Lakatos (1976) is that translating a mathematical argument into a more formal idiom transforms it. By the time it is fully formalised (satisfying criteria (a) and (b) above), it is no longer the same piece of reasoning. ${ }^{15}$ Such

14 Note that operations that seem impossible in a domain may become possible as a result of mathematical developments. See Larvor (2010).

15 See p. 116, where Epsilon's translation turns the theorem about polyhedra into a theorem about the dimensions of vector spaces, 99105 ("How criticism may turn Mathematical Truth into Logical Truth") and 120-123, where the class discusses translation. 
translations are not 'routine' (to pick up Mac Lane's word); rather, traduttore, traditore. Since then, Rav has argued with a wealth of examples that proof-ideas are among the most interesting mathematical ideas (Rav 1999). In his view, what gets washed out in the process of radical formalisation is most of the mathematics. Thinking of proofs as recipes for creating derivations obscures this fact, because this approach requires us to regard the mathematical proof-ideas as mere heuristics. Avigad (in Mancosu 2008) argues with examples that understanding proofs does not consist in seeing how a suitable derivation could be manufactured. Articles by Goethe \& Friend, Nickel, Pelc and Thurston make versions of the same claim. It is true that proofs written by experts for other experts are highly compressed. They omit steps that a 'properly instructed' expert reader can be expected to reconstruct. In this sense, published expert-level proofs are argument-recipes. However, they are not recipes for creating derivations in the proof-theoretic sense. To see this, observe that in filling in the gaps, an expert reader does not normally do the things required to translate a proof-used-in-earnest into a derivation satisfying (a) and (b). In working over a proof, one does not normally specify a formal language and a set of inference rules. On the contrary, we use minor, inessential abuses of notation to keep the page free from unhelpful clutter. McLarty ${ }^{16}$ points out that mathematicians routinely conflate terms that formal treatments would distinguish (such as identifying the real number $x$ with the complex number $x+0 i$ ). If McLarty's argument is correct, then formalisation does not only discard or distort the original mathematical content; it also introduces irrelevancies such as the distinction between $\mathbf{R}$ and its image in $\mathbf{C}$. Sometimes, we use highly general inference-licences that are not recognised in standard formal systems, such as symmetry considerations. ${ }^{17}$

16 McLarty (2008 p. 357)

17 Barwise (1989) p. 849; for discussion see Dawson p. 270. Barwise reports Kreisel's remark that, " $99 \%$ of all mathematicians don't know the rules of even one of these formal systems, but still manage to give correct proofs." (ibid). 
Of course, the expert reader's version - the published proof plus some gap-filling workings-out-is still highly compressed. To continue the process, imagine a less expert reader, who has to be shown in more detail why each step follows from what has gone before. Translating the proof into a fully formal idiom would not help this person at all. If the gap-filling process has a limit (on which see Rav 1999:14 15), it would be the version one would have to give to a person with no prior mathematical understanding at all. Far from having all the non-logical meanings washed out, this proof would have to explicate all the mathematical concepts that the proof invokes. Such explication cannot consist in replacing mathematical terms with their formal definitions. To see why, consider the definition of a continuous real-valued function. The formal version reads: for any real $\varepsilon$ there is a $\delta$ such that... The vital ingredient, the part that explains to the student why this is a definition of continuity, is missing. The version the student needs in order to understand the mathematics reads: for any real $\varepsilon$, however small, there is a $\delta$ such that... The vital mathematical thought, that there is a suitable $\delta$ for arbitrarily small $\varepsilon$, is not 'buried' in the fully formal version, in the sense of being spread over too many pages or obscured by thickets of notation. It is absent from the formalised version. But it is precisely in grasping such thoughts that one understands a proof, and it is prior understanding of such thoughts that allows expert mathematicians to read the highly compressed proofs in mathematics journals. Such is the common stock of the philosophy of mathematical practice.

As noted at the outset, this line of argument leaves the opposition with easy replies. First, many of the points raised by philosophers of mathematical practice concern understanding rather than the primary role of proofs, namely, securing the truth of theorems. The phrase 'however small' is absent from formal proofs in real analysis because the formal proof goes through without it. As is often the case in philosophy, the disputants end up begging the questions against each other. The philosopher of mathematical practice will insist that it is the informal, content-dependent proof that 
does the proving; the opposition will reply that it does so only because it somehow specifies or indicates a formal (i.e. content independent) derivation. Practice-oriented philosophers can stall for a while by pointing out that these derivations are rarely if ever seen in practice. Therefore, if anything proves theorems, it must be the 'real' proofs. However, philosophers of practice must eventually substantiate this claim by explaining how these proofs prove. This requires a development of logical theory.

\section{Proofs as Systems of Inferential Actions}

At the very least, the explanatory task requires a conception of inference that is broad enough to include the moves that mathematicians make in the course of such proofs, which is what the phrase 'inferential action' is intended to permit. Many of these moves are not actions on propositions. This is most obvious in the case of manipulations of diagrams, mental images or physical models, such as the simple proof that the composition of knots is commutative (tie a thumb-knot in a piece of actual or mental string; notice that you can shuffle the knot along the string; tie another knot on the same string, but loosely; notice that you can shuffle the tighter knot all the way round the looser

knot until it ends up on the other side). ${ }^{18}$ Or consider J.W.H. Alexander's 1923 proof that every knot can be represented as a closed braid. To prove this, it is enough to show that every knot can be manipulated so that it is 'coiled' around a point, the braid axis. In a coiled knot, a path along the string always goes the same way round the braid axis (clockwise or anticlockwise). The core of the proof is this: if the knot in hand has a section that goes the wrong way round the braid axis, 'throw it over your shoulder' (that is, flip it to the opposite side of the braid axis). Demonstrating one such

18 Sossinsky (2002:49) 
throw with a piece of actual string or a chalkboard diagram (rubbing out the flipped section and redrawing it in its new position) is the core inferential act of the proof. ${ }^{19}$

For a more sophisticated case from the same area of mathematics, consider the operation of 'combing-out' employed in Churchard and Spring (1988). To see the unnaturalness of thinking of their proof as a series of operations on propositions, consider this passage, in which they explain how to extend their principal result:

The above may be extended to more general target manifolds other than $\mathbf{R}^{P}$ when we look at the "combing out" procedure in terms of vector fields. Consider the special case $f: \mathbf{R}^{1} \rightarrow \mathbf{R}^{3}$ and the radial vector field $V$ on $\mathbf{R}^{3}$ given by $V(p)=p, p \in \mathbf{R}^{3}$. To show the above proposition, we could have isotoped $f$ near the origin in $\mathbf{R}^{1}$ to lie along the paths of two trajectories of $V$ emanating from $0 \in \mathbf{R}^{3}$ (e.g., the $x$-axis). Then using the flow of $V, f$ could be "combed out" along these trajectories. In this fashion, all the "knotted" behaviour of $f$ is swept out to $\infty$ by a proper isotopy, leaving behind the inclusion $\mathbf{R}^{1} \rightarrow \mathbf{R}^{3}$. (1988:138)

Aside from referring to the 'combing-out procedure' and sweeping out the knotted behaviour of $f$, they cheerfully turn 'isotope' into a verb—-with $f$ as its object. Other proof-procedures that are not naturally described as actions on propositions include the construction of numbers, functions,

19 Op. cit. pp. 17-20. I owe this example to a conversation with David Corfield. See Jones (1997:209-213). Here I must record a mild terminological disagreement with Goethe and Friend (2010), who describe a derivation from axioms as the 'core' of a proof (p. 277). Given their argument, this is odd. In the example they offer (the proof that the rational numbers are equinumerous with the natural numbers), the core of the proof is the act of drawing a path through the grid of rationals — which does not appear in the fully formalised version. 
mappings and structures of every sort; artful indexing (think of Gödel numbering); and any instruction beginning "let", "set" or "take".

As noted above, for every kind of inferential action, there must be a corresponding means of control, to ensure rigour. ${ }^{20}$ Sometimes these controls are simple rules like 'do not divide by zero'. In other cases, these controls may be the fruit of mathematical research (think of the seventeenthcentury experiments in exponentiation, or the nineteenth-century developments necessary to establish rules for handling infinite series). In some historical cases the controls are implicit, and there the historian-philosopher has a task to perform in teasing them out explicitly (Manders' work on Euclid (1995) is a model of this). Such teasings-out are a job for a philosopher-historian rather than a historian tout court because the outcome must be an explanation of how successful inferences were possible, that is, a logical account (in our broad sense of 'logic' as the study of inferential acts). Demonstrating rigour involves making the controls on inferential acts explicit, which is why some diagrams disappear from the final published version of a mathematical argument. The problem is not with diagrams as such, but rather that the actions performed on these diagrams in this piece of work do not have established, agreed controls. ${ }^{21}$

A related task for the philosopher is to understand the relationship between inferential actions on non-propositional objects and the frame that turns a gesture into an inference. There must be such a frame, even if it too is largely or wholly implicit. Dropping a weight from a height is not an experiment unless it is framed as such. Even performing a new gymnastic feat does not become an argument without a framing phrase such as, "Watch this!", or a gesture with the same illocutionary

\footnotetext{
20 "At its most basic, a mathematical practice is a structure for cooperative effort in the control of self and life... Successes of control may be seen in the way we can expect the world to behave..." (Manders 1995:82; emphasis in original).

${ }^{21}$ See Carter (2010) for a case of disappearing diagrams.
} 
force (recall the pointed watch-staring in the earlier example of dropping a stone down a well). The fact that such frames are usually linguistic or textual does not reduce inferential acts to speech-acts. A diagram combines with a text to constitute an argument, but the diagram does not thereby become a kind of text. ${ }^{22}$ A manipulation of physical matter becomes an experiment when combined with the discursive practice of writing-up and publication; this does not show that the manipulation of matter is a kind of discourse. Inferential acts and speech-acts are overlapping categories, but neither one wholly contains the other.

The notion of inferential action offered here is no more than a sketch, but, given the diversity of domains and the inventiveness of inferring agents, there can never be a complete taxonomy of inferential actions, let alone a comprehensive theoretical treatment. Nevertheless, this sketch does set out practical tasks (the identification of inferential actions and their objects; the identification of their associated systems of control and rigour; the explication of their linguistic frames) that could constitute one form of normal research in the philosophy of mathematical practice; the fruits of such work could in turn provide readings of informal proofs that show how they work as proofs without invoking non-existent formal derivations.

\section{Acknowledgements}

I am grateful to symposiasts at Nancy, Leicester, Amsterdam, Crewe and Canterbury for their polite attention to and helpful questions regarding ancestors of this paper, and to two anonymous referees for their invaluable suggestions.

\footnotetext{
${ }^{22}$ In addition to Manders' work on Euclid, See Dove (2002) for a careful account of how a diagram functions in proofs that does not assimilate diagrams to text or reduce them to illustration or heuristics. See also Grosholz (2007) and Reed (1995).
} 


\section{References}

Aberdein, Andrew (2006) 'The Informal Logic of Mathematical Proof' in Hersh (2006) pp. 56-70

Aigner, Martin \& Ziegler, Günter M. (2004) Proofs from The Book. Springer (3 ${ }^{\text {rd }}$ edition).

Aspray, W. and Kitcher, P. [1988] History and Philosophy of Modern Mathematics. (Minnesota Studies in the Philosophy of Science, 11) Minneapolis, University of Minnesota Press.

Avigad, Jeremy (2008) 'Understanding Proofs' in Mancosu (2008) pp. 317-353.

Azzouni, Jody (2006) 'How and Why Mathematics is Unique as a Social Practice' in Hersh (ed.) 2006.

Baker, Alan (2007) 'Is there a problem of induction for mathematics?' in Leng et al. (eds).

Barwise, Jon, (1989) 'Computers and Mathematics'. Notices of the American Mathematical Society 36 (7): 844-851.

Beardsley, Monroe C. (1950) Practical Logic. Prentice-Hall: New Jersey.

Buldt, B., Löwe, B., \& Müller, T. [2008] Towards a New Epistemology of Mathematics. Special edition of Erkenntnis 68/3

CadwalladerOlsker, Todd (2011) 'What Do We Mean by Mathematical Proof?' The Journal of Humanistic Mathematics, 1 (1): 33-60.

Carter, Jessica (2010) 'Diagrams and Proofs in Analysis'. International Studies in the Philosophy of Science 24 (1):1-14.

Cellucci, C. \& Gillies, D. [2005] Mathematical Reasoning and Heuristics. London: King's College Publications.

Churchard, Peter and Spring, David (1988) 'Proper Knot Theory in Open 3-Manifolds' Transactions of the American Mathematical Society, Vol. 308, No. 1, pp.133-142.

Corfield, David (2003) Towards a Philosophy of Real Mathematics, Cambridge University Press.

Dawson, John W. Jr. (2006) 'Why Do Mathematicians Re-prove Theorems?' Philosophia Mathematica 14(3): 269-286.

Dove, Ian (2002) ‘Can pictures prove?' Logique et Analyse vol. 179-180 pp. 309-340.

Eemeren, F.H. van, \& Grootendorst, R. (2004). A systematic theory of argumentation: The pragmadialectical approach. Cambridge: Cambridge University Press. 
Fisher, Alec (2004) The Logic of Real Arguments. Cambridge University Press (second edition; first edition 1988).

Fogelin, Robert J. and Sinnott-Armstrong, Walter (2001) Understanding Arguments. Harcourt College Publishers ( $6^{\text {th }}$ edition).

Galison, Peter (1987) How Experiments End. Chicago: Chicago University Press.

Galison, Peter (1997) Image and Logic: a Material Culture of Microphysics. Chicago: Chicago University Press.

Goethe, Norma \& Friend, Michèle (2010) 'Confronting the Ideals of Proof with the Ways of Proving of the Research Mathematician'. Studia Logica 96:273-288.

Gowers, W.T. (2007) 'Mathematics, Memory and Mental Arithmetic' in Leng et al. (eds)

Grosholz, E. \& Breger, H. [2000] The Growth of Mathematical Knowledge (Synthese Library volume 289). Kluwer.

Grosholz, Emily (2007) Representation and Productive Ambiguity in Mathematics and the Sciences. Oxford: Oxford University Press.

Groarke, Leo (1999) 'The Pragma-Dialectics of Visual Argument' in Proceedings of the Fourth International Conference of the International Society for the Study of Argumentation. van Eemeren, Grootendorst, Blair \& Willard (eds). Amsterdam. pp. 276-280.

Hanna, G., Jahnke, H. \& Pulte, H. [2010] Explanation and Proof in Mathematics: Philosophical and Educational Perspectives. Springer.

Hersh, Reuben (ed.) (2006) 18 Unconventional Essays on the Nature of Mathematics. Springer.

Hoagland, John (1999) 'Informal Logic: The Two Schools' in Proceedings of the Fourth International Conference of the International Society for the Study of Argumentation. van Eemeren, Grootendorst, Blair \& Willard (eds). Amsterdam. pp. 348-352.

Johansen, Mikkel Willum (2010) 'Embodied strategies in mathematical cognition' in Löwe and Müller (eds) PhMSAMP Philosophy of Mathematics: Sociological Aspects and Mathematical Practices. London: College Publications. Texts in Philosophy11: pp. 179-196.

Jones, V.F.R. (1998) 'A Credo of Sorts' in Dales \& Oliveri (eds) Truth in Mathematics. Oxford University Press. Pp. 203-214.

Lakatos, Imre (1976) Proofs and Refutations. Cambridge: CUP. Worrall \& Zahar (eds). 
Larvor, Brendan (2010) 'Syntactic Analogies and Impossible Extensions' in Löwe, B., \& Müller, T. PhiMSAMP. Philosophy of Mathematics: Sociological Aspects and Mathematical Practice (Texts in Philosophy volume 11). College Publications.

Leng, M., Paseau. A., and Potter, M., (eds) (2007) Mathematical Knowledge. Oxford: Oxford University Press.

Levi, Don (2010) “Against the logicians' The Philosophers' Magazine 51: 80-86.

Löwe, Benedikt and Müller, Thomas (2010) Philosophy of Mathematics: Sociological Aspects and Mathematical Practices. London: College Publications.

Mac Lane, Saunders (1986) Mathematics: Form and Function. Springer.

Mancosu, Paolo (ed.) (2008) The Philosophy of Mathematical Practice. Oxford: Oxford University Press.

Manders, Ken (1995) 'The Euclidean Diagram' in Mancosu (2008) pp. 80-133.

McLarty, Colin (2008) 'What Structuralism Achieves' in Mancosu (2008) pp. 354-369.

Nabonnand, P. [2005]. Fonder Autrement les Mathématiques. Paris: Kimé. Special issue of Philosophia Scientiae.

Nickel, Gregor (2010) 'Proof: Some notes on a phenomenon between freedom and enforcement' in Löwe and Müller (2010) pp. 281-291.

Pelc, Andrzej (2009) 'Why do we believe theorems?' Philosophia Mathematica 17(1): 84-94.

Rav, Yehuda (1999) 'Why Do We Prove Theorems?' Philosophia Mathematica 7: 5-41.

Reed, David (1995) Figures of Thought. London and New York: Routledge.

Robinson, J.A. (2000) 'Proof = Guarantee + Explanation' in Intellectics and Computational Logic: Papers in Honor of Wolfgang Bibel. Dordrecht: Kluwer.

Scriven, Michael (1976) Reasoning. McGraw-Hill.

Sossinsky, Alex (2002) Knots: Mathematics With a Twist. Cambridge, (Mass.); London (UK): Harvard University Press.

Thurston, William P. (2006) 'On Proof and Progress in Mathematics' in Hersh (2006) pp. 37-55.

Walton, Douglas, Reed, Chris and Macagno, Fabrizio (2008) Argumentation Schemes. Cambridge: Cambridge University Press. 
Tymoczko, T. [1998] New Directions in the Philosophy of Mathematics. Basel and Boston, Birkhäuser. Revised and expanded edition in Princeton University Press.

Van Kerkhove, B.; Van Bendegem, J.-P. [2002] Perspectives on Mathematical Practices. Special issue of Logique et Analyse. Vol.45 No.179-180

Van Kerkhove, B.; Van Bendegem, J.-P. [2007] Perspectives on Mathematical Practices: Bringing Together Philosophy of Mathematics, Sociology of Mathematics, and Mathematics Education. Series: Logic, Epistemology, and the Unity of Science, Vol. 5. Dordrecht: Springer.

Van Kerkhove, B.; Van Bendegem, J.-P. (2009) 'Mathematical Arguments in Context'. Foundations of Science vol. 14 numbers 1 and 2. pp. 45-57.

Van Kerkhove, B. [2009] New Perspectives on Mathematical Practices. World Scientific Publishing Company. Series: Texts in Philosophy 11. World Scientific Publishing. Walton, Douglas (1998) The New Dialectic; Conversational contexts of argument. Toronto University Press; Toronto.

Whitehead, A.N. \& Russell, B. (1962) Principia Mathematica to *56. Cambridge University Press. (Abridgement of 1927 second edition) 